\title{
Expression of maspin in endometrioid adenocarcinoma of endometrium
}

\author{
HANG WUN RAYMOND LI ${ }^{1}$, SEE WAI LEUNG ${ }^{1}$, CHUN SING SAMUEL CHAN ${ }^{1}$, \\ MAY MEI-YUNG YU ${ }^{2}$ and YICK FU WONG ${ }^{1}$ \\ Departments of ${ }^{1}$ Obstetrics and Gynaecology, and ${ }^{2}$ Anatomical and Cellular Pathology, \\ The Chinese University of Hong Kong, Prince of Wales Hospital, Shatin, New Territories, Hong Kong, P.R. China
}

Received March 16, 2006; Accepted May 30, 2006

\begin{abstract}
Maspin is a member of the serpin family, whose expression is altered in neoplasia and malignancies of many tissues. Underexpression of maspin has been reported in breast and prostatic cancers, but in some cancers such as ovarian, colorectal and pancreatic carcinoma, it was found to be upregulated. This study aimed at demonstrating the expression of maspin in human endometrial tissue and searching for any altered expression in endometrioid adenocarcinoma of the endometrium compared to normal endometrium. The expression level of the maspin gene was studied using reverse transcriptase-polymerase chain reaction (RT-PCR) performed on RNA extracted from 34 endometrial cancer samples (including 24 with FIGO stage I disease and 10 with FIGO stage III disease) and 28 normal endometrium in proliferative or secretory phases. Immunohistochemical staining was also performed on 10 cases of endometrial cancer (6 FIGO stage I cases and 4 FIGO stage III cases) as well as 15 normal endometrium. Semi-quantitative RT-PCR revealed that the expression of maspin was significantly up-regulated in both stage I $(\mathrm{p}<0.01)$ and stage III $(\mathrm{p}<0.01)$ endometrial cancer compared with normal endometrium. However, no significant difference in maspin expression was demonstrated between stage I and stage III endometrial cancer. Immunostaining of all tissue sections revealed an immunopositive signal in the nuclei of the normal or cancerous endometrial glandular cells. In $60 \%$ of the cancer cases, cytoplasmic staining was also evident. Our results suggested that there is up-regulated expression of maspin in endometrioid endometrial adenocarcinoma. Cytoplasmic immuno-expression of maspin is common in endometrial cancer. It may play a role in the malignant transformation of human endometrial tissue.
\end{abstract}

Correspondence to: Dr H.W.R. Li, Department of Obstetrics and Gynaecology, Prince of Wales Hospital, Shatin, New Territories, Hong Kong, P.R. China

E-mail: raymondli@hkam.org.hk

Key words: endometrium, endometrioid adenocarcinoma, maspin

\section{Introduction}

Maspin (mammary serine protease inhibitor) is a tumour suppressor gene which belongs to the serpin family. The gene is mapped to chromosome $18 \mathrm{q} 21.3$. It encodes a $3.0-\mathrm{kb}$ mRNA transcript that translates into a $42-\mathrm{kDa}$ protein (1). It has been shown to have tumour-suppressing function by its inhibitory actions on motility, invasion, metastasis and angiogenesis in human breast and prostatic cancers $(1,2)$. Maspin was originally identified from mammary epithelium by subtractive hybridization in normal mammary epithelium and mammary cancer tissue (3).

It has been demonstrated that maspin is present in the epithelium of several normal human organs (such as prostate, thymus, testis, small intestine, and colon) and particularly in the myoepithelium of breast tissue, in which it is predominant. However, it is down-regulated during cancer progression. Its expression decreases with increasing levels of malignant behaviour. It also functions as an inhibitor of angiogenesis. Its down-regulation is regulated at the transcription level. The re-expression of maspin by pharmacological intervention may also be a potential therapeutic option (1-4).

However, other findings showed that maspin was paradoxically overexpressed in pancreatic cancers (5) and ovarian cancers (6), whereas it is not or minimally expressed in normal pancreatic and ovarian epithelium.

To date, there are no published reports in the literature evaluating the expression and role of maspin in endometrial cancer. Thus, we conducted this study to investigate the expression of maspin in endometrial cancer in comparison to normal endometrium. We also studied the correlation between maspin expression and the clinical staging of the disease, which might be of prognostic significance. We limited our study only to endometrioid adenocarcinoma, which is the most common histological subtype of endometrial cancer.

\section{Materials and methods}

We studied the expression of maspin using reverse transcriptase-polymerase chain reaction (RT-PCR) and immunohistochemistry.

Specimen collection. Frozen tissue sections on 34 cases of endometrioid endometrial adenocarcinoma (24 FIGO stage I 
Table I. Primers and PCR conditions used in the semi-quantitative RT-PCR analysis.

A, Primers used.

\begin{tabular}{llcr}
\hline Gene product & \multicolumn{1}{c}{ Primers } & Product size (bp) & Reference \\
\hline Maspin & Forward 5'-CCA CAG GCT TGG AGA AGA TTG A-3' & 338 \\
& Reverse 5'-GGT CAG CAT TCA ATT CAT CCT TGT-3' & 6 \\
18S rRNA & Forward 5'-GTA ACC CGT TGA ACC CCA TT-3' & 131 \\
& Reverse 5'-CCA TCC AAT CGG TAG TAG CG-3' & 22 \\
\hline
\end{tabular}

$\mathrm{B}, \mathrm{PCR}$ conditions.

\begin{tabular}{|c|c|c|c|c|c|c|c|}
\hline $\begin{array}{l}\text { Gene } \\
\text { product }\end{array}$ & $\begin{array}{c}\text { Cycle } \\
\text { conditions }\end{array}$ & $\begin{array}{l}\text { No. of } \\
\text { cycles }\end{array}$ & $\begin{array}{c}\text { Final } \\
\text { extension }\end{array}$ & $\begin{array}{c}\mathrm{MgCl}_{2} \\
\text { concentration } \\
(\mu \mathrm{M})^{\mathrm{a}}\end{array}$ & $\begin{array}{c}\text { Primer } \\
\text { concentration } \\
(\mu \mathrm{M})^{\mathrm{a}}\end{array}$ & $\begin{array}{c}\text { Taq DNA } \\
\text { polymerase } \\
\text { (Units) }^{\mathrm{a}}\end{array}$ & $\begin{array}{c}\mathrm{dNTP} \\
\text { concentration } \\
(\mu \mathrm{M})^{\mathrm{a}}\end{array}$ \\
\hline Maspin & $\begin{array}{l}94^{\circ} \mathrm{C} \times 60 \mathrm{sec} \\
60^{\circ} \mathrm{C} \times 150 \mathrm{sec} \\
72^{\circ} \mathrm{C} \times 60 \mathrm{sec}\end{array}$ & 40 & $72^{\circ} \mathrm{C} \times 4 \mathrm{~min}$ & 1.5 & 0.4 & 0.8 & 0.2 \\
\hline $18 \mathrm{~S}$ rRNA & $\begin{array}{l}94^{\circ} \mathrm{C} \times 20 \mathrm{sec} \\
62^{\circ} \mathrm{C} \times 30 \mathrm{sec} \\
72^{\circ} \mathrm{C} \times 30 \mathrm{sec}\end{array}$ & 35 & $72^{\circ} \mathrm{C} \times 4 \mathrm{~min}$ & 2.0 & 2.0 & 1.0 & 0.2 \\
\hline
\end{tabular}

${ }^{\mathrm{a} C o n c e n t r a t i o n s ~ o f ~ r e a g e n t s ~ i n ~ a ~ f i n a l ~ r e a c t i o n ~ v o l u m e ~ o f ~} 20 \mu 1$. Each reaction mixture also contained $2 \mu 1$ of $10 \mathrm{X}$ PCR buffer and $2 \mu 1$ of the respective cDNA sample.

cases and 10 FIGO stage III cases) and 28 cases of normal endometrium (including both proliferative and secretory endometrium but excluding atrophic endometrium) were collected and prepared at the Department of Obstetrics and Gynaecology, Prince of Wales Hospital, Hong Kong. Formalin-fixed paraffinembedded tissues of normal endometrium and endometrial carcinoma were also collected from the Department of Obstetrics and Gynaecology and the Department of Anatomical and Cellular Pathology, Prince of Wales Hospital. They included 10 cases of endometrioid endometrial adenocarcinoma (6 FIGO stage I cases and 4 FIGO stage III cases) and 15 normal endometrium including 5 cases each of proliferative endometrium, secretory endometrium and atrophic endometrium. The histological diagnosis in all cases was confirmed by a qualified gynaecological pathologist (M.M.Y.Y.) and the clinical information including FIGO staging was retrieved from the patients' clinical records. The research protocol was approved by the Clinical Research Ethics Committee, The Chinese University of Hong Kong. Informed consent was obtained from the human subjects from whom tissue samples were collected.

$R T$-PCR. Manual micro-dissection was performed on the frozen tissue sections to obtain purified endometrial glandular tissue. Total cellular RNA was extracted using the RNeasy mini kit (Qiagen Inc., CA, USA) according to the manufacturer's protocol. From each case, $1 \mu \mathrm{g}$ of extracted RNA was subjected to reverse transcription using the SuperScript II reverse transcriptase kit (Invitrogen Corporation, CA, USA).

A cDNA (1 $\mu 1)$ sample synthesized from each case was then amplified by polymerase chain reaction (PCR) using specific primers and PCR conditions as listed in Table I. Normal human breast tissue cDNA was used as positive control. Negative control was set up by replacing the cDNA sample with water. The PCR products were electrophoresed on $2 \%$ agarose gels stained with ethidium bromide, and analyzed using the Gel Doc 1000 system (Bio-Rad Laboratories, CA, USA) with the band intensities quantitated in an arbitrary unit. The 18S rRNA housekeeping gene was used for normalization of RNA quantity in each case.

Immunohistochemistry. Serial 5-mm sections were made from the formalin-fixed paraffin-embedded tissue. The sections were de-waxed in xylene and rehydrated through graded alcohol and then in tris-buffered saline (TBS). Endogenous peroxidase was blocked using $3 \%$ hydrogen peroxide for $5 \mathrm{~min}$. For antigen retrieval, the sections were heated to boil in a microwave oven in $0.01 \mathrm{M}$ sodium citrate buffer and cooled to room temperature. Non-specific binding was blocked by incubating the sections with Protein Block Serum-Free (Dako Corp., CA, USA) for $15 \mathrm{~min}$. Immunohistochemistry was performed using a mouse anti-human maspin antibody (Pharmingen, San Diego, CA, USA). The optimal antibody dilution was determined to be 1:200 in a preliminary trial experiment. After incubation with the primary antibody for $90 \mathrm{~min}$, the sections were then incubated with a secondary link antibody followed by peroxidase-conjugated streptavidin (Dako LSAB+ kit; Dako Corp.) for 20 min each. TBS was used as the wash buffer. The peroxidase reaction was performed by incubating with diaminobenzidine-hydrogen peroxide (Liquid DAB-Plus substrate kit; Zymed, CA, USA) for $5 \mathrm{~min}$. The sections were lightly counterstained with haematoxylin, dehydrated in graded alcohol, cleared in xylene, and mounted.

The sections were examined under medium (x200) and high power $(\mathrm{x} 400)$ light microscope. Assessment of maspin immunostaining was semi-quantitative and was scored with 


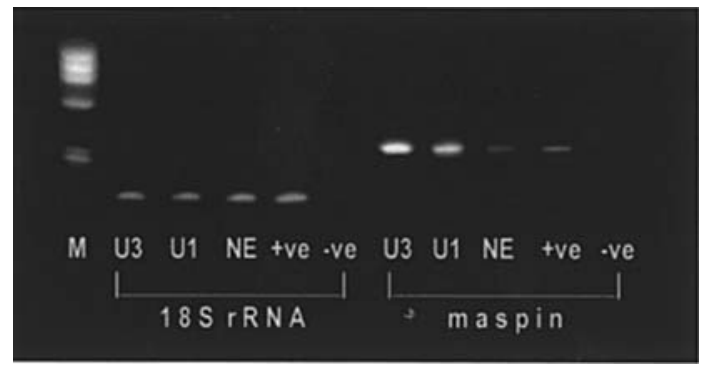

Figure 1. Representative results from semi-quantitative RT-PCR analysis of maspin in normal endometrium (NE), stage I endometrial cancer (U1) and stage III endometrial cancer (U3). The housekeeping gene 18S rRNA was used as internal control. Up-regulated transcription of maspin was observed in both stage I and stage III endometrial cancer compared to normal endometrium. (+ve, positive control using human breast tissue cDNA; -ve, negative control by omitting cDNA during PCR; M, DNA marker ladder.)

respect to relative intensity on an arbitary scale ( 0 , no staining; 1, weak; 2 , moderate; and 3 , intense) as well as the percentage of immuno-positive cells estimated and graded as follows: $0,0-25 \% ; 1,26-50 \% ; 2,50-75 \%$; and $3,76-100 \%$. The immunoreactive score for each case was the sum of the two parameters, which ranged from 0 to 6 .

Sections of normal human mammary tissue were used as positive control. Negative controls were set up by substituting the primary antibody with TBS.

Statistical analysis. Comparison of the immunostaining scores and the band intensities from gel electrophoresis of the RT-PCR products between normal and cancer groups were analyzed by the Kruskal-Wallis test with Dunn's post-hoc analysis (for multiple group comparisons) and Mann-Whitney U test (for two-group comparisons). All analyses were performed by GraphPad Prism 4 for Windows (GraphPad Software, San Diego, California, USA). A p-value of $<0.05$ was considered as statistically significant.

\section{Results}

Semi-quantitative $R T$-PCR. The results for 28 normal endometrium (including both proliferative and secretory endometrium, but excluding atrophic endometrium) and 34 endometrial cancer (including 24 FIGO stage I cases and 10 FIGO stage III cases) were analysed. A significantly increased expression of maspin in endometrial cancer compared to normal endometrium was demonstrated by semi-quantitative RT-PCR analysis ( $\mathrm{p}=0.0003$, Kruskal-Wallis test). One set of representative results is shown in Fig. 1. Dunn's post-hoc test revealed that maspin expression in both stage $\mathrm{I}(\mathrm{p}<0.01)$ and stage III $(p<0.01)$ cancers was significantly higher compared to normal endometrium, but there was no significant difference in maspin expression between stage I and stage III endometrial cancers ( $p>0.05)$. The data are presented in a graph (Fig. 2).

Further analysis was made of maspin expression in welldifferentiated (grade I) and moderately/poorly-differentiated (grade II and III) endometrial cancer, but no significant difference was demonstrated ( $\mathrm{p}>0.05$, Mann-Whitney U test). Among the normal endometrium cases, maspin expression in proliferative and secretory endometrium was also compared,

\section{Semi-quantitative RT-PCR} Results

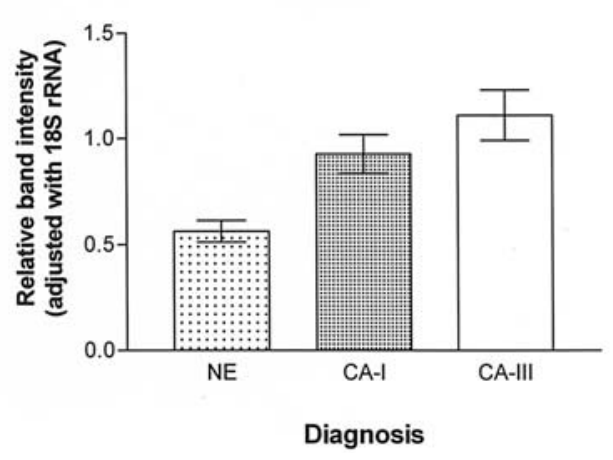

Figure 2. Semi-quantitative RT-PCR analysis of maspin expression in endometrial cancer and normal endometrium (NE). The relative band intensity of each gene was quantified as ratios to 18S rRNA. The bars show the mean and SEM of each respective group. Significantly higher expression of maspin was found in both FIGO stage I endometrial cancers (CA-I) $(\mathrm{p}<0.01)$ and FIGO stage III endometrial cancers (CA-III) $(\mathrm{p}<0.01)$ compared to normal endometrium. There is no significant difference in maspin expression between CA-I and CA-III ( $p>0.05)$.

and again no significance difference was found $(\mathrm{p}>0.05$, Mann-Whitney U test).

Immunohistochemistry. In normal endometrial tissue, expression of maspin was localized to the nuclei of the glandular epithelial cells. Some of the stromal mesenchymal cells were also positively stained. The level of staining was similar in proliferative endometrium and secretory endometrium, but there was reduced immunostaining in atrophic endometrium (Fig. 3).

In endometrial cancer, positive immunostaining for maspin was demonstrated in the nuclei of endometrial carcinoma cells in the majority of cases. In 6 out of the 10 cases, concurrent distinct cytoplasmic staining was also found (Fig. 4).

The immunostaining signal was semi-quantitated by the immunoreactive score. There was no significant difference demonstrated in the immunoreactive score between normal endometrium and endometrial cancer ( $\mathrm{p}>0.05$, Mann-Whitney $\mathrm{U}$ test), but this study was not adequately powered to demonstrate such a difference, if any, due to the limited sample size.

\section{Discussions}

Maspin was a gene originally identified in normal breast epithelial tissue. Its expression was found to be down-regulated in cancers of the breast and prostate, where it functions as a tumour suppressor gene and inhibits angiogenesis, tumour cell motility and invasion, enhances cell adhesion, and suppresses tumour progression and metastasis $(2,4)$. Our recent study has also shown down-regulated expression of maspin in gestational trophoblastic diseases (7). However, there were also reports that up-regulation of maspin was associated with tumour progression in breast cancer (8-10). On the contrary, overexpression of maspin has been demonstrated in cancers of some other tissues such as pancreas $(5,11)$, ovary (6), stomach (12), colorectum (13), thyroid (14), lung $(15,16)$, and larynx (17), where it may function as an oncogene instead. 

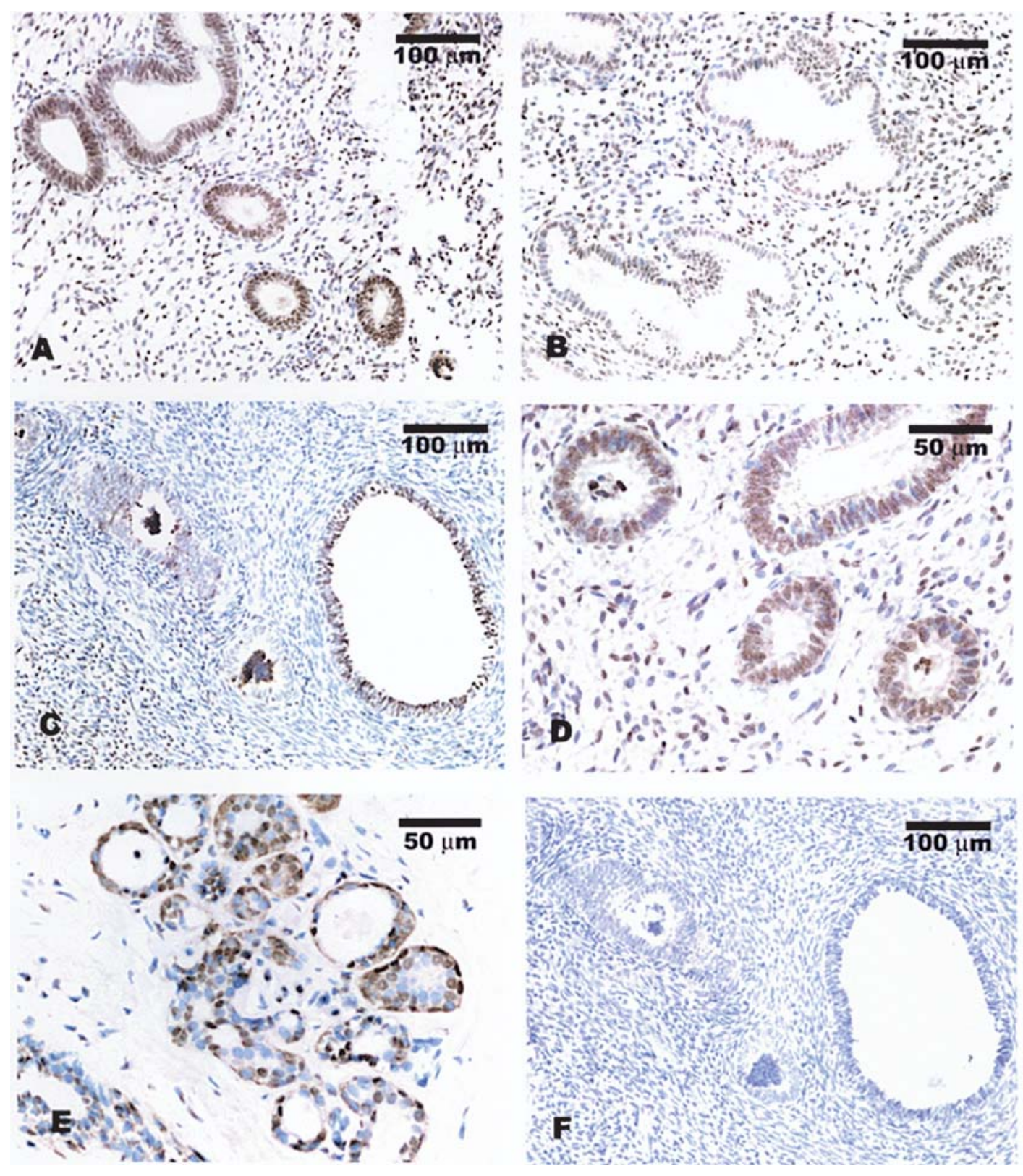

Figure 3. Immunohistochemical staining for maspin in normal endometrial tissue. A, proliferative phase endometrium (medium power); B, secretory phase endometrium (medium power); $\mathrm{C}$, atrophic endometrium (medium power); $\mathrm{D}$, proliferative phase endometrium (high power); $\mathrm{E}$, human breast tissue as positive control; F, normal endometrial tissue with omission of primary antibody as negative control. In normal endometrial tissue, nuclear staining is found in the endometrial glandular cells. Some of the stromal mesenchymal cells are also stained.
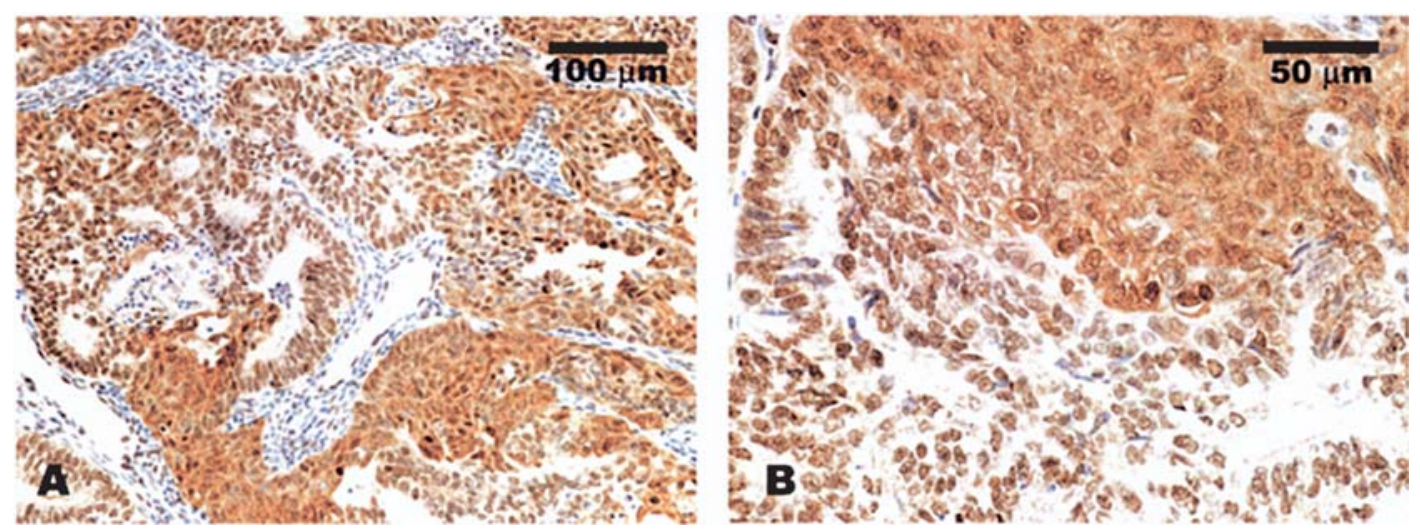

Figure 4. Immunohistochemical staining for maspin in endometrioid endometrial adenocarcinoma. Photomicrograph of a representative case is shown. A, medium power; B, high power. Concurrent nuclear and cytoplasmic staining are both shown in the cancerous cells. 
To our knowledge, our study (7) was the first report in the literature on the expression of maspin in endometrial cancer. We speculated that maspin gene alteration might be involved in the pathogenesis of endometrial cancer because the biological behaviour of endometrial cancer resembles that of breast cancer in many ways. Moreover, the maspin gene is mapped to chromosome $18 \mathrm{q} 21.3$ in close proximity to the DCC and DPC4 genes, a region of frequent loss of heterozygosity in endometrial cancer (18). By RT-PCR, we demonstrated an up-regulated expression of maspin in both early- and late-stage endometrial cancers compared to normal endometrial tissue. Significant difference was not demonstrated in the immunohistochemical sections, probably because of the small sample size which limited the power of statistical analysis. Nonetheless, the results from the RT-PCR did infer a significant up-regulation of the maspin gene transcription in endometrial cancer. Despite the small sample size, this study did serve as an important preliminary work that would prompt further investigations into its pathogenetic role as well as prognostic significance in endometrial cancer.

The main purpose of the immunostaining in this study was to localize the expression of maspin in normal and cancerous endometrial tissue. Nuclear expression was demonstrated in glandular epithelial cells of normal endometrium, whereas concurrent cytoplasmic expression was demonstrated in $60 \%$ of cases of endometrial cancer. This finding did agree with most previous reports that, in cancers where maspin is up-regulated, nuclear localization is usually associated with better prognosis, whereas cytoplasmic localzation is usually associated with more malignant behaviour or poor clinical prognosis (16). The precise molecular and biological mechanism of maspin is still not clearly understood at present, although the role of subcellular localization of maspin in influencing its function in carcinogenesis has been repeatedly speculated. This functional regulation of maspin at the level of subcellular localization may explain the apparently paradoxical findings of either up-regulation or down-regulation in different cancers. It has been postulated that tumour suppression, the normal function of maspin, is associated with the nuclear-localised maspin, whereas cytoplasmic maspin may be the biologically inactive form resulting from the auto-inhibition of its activity by polymerization. Thus, an absolute decrease in nuclear expression of maspin, or a relative decrease in the nuclear/cytoplasmic expression ratio may promote carcinogenesis (15-17). A study of the functional assays of maspin activity, which does not currently exist, is required to delineate the mechanism.

With regard to the underlying molecular action of maspin in cell invasion and carcinogenesis, it was proposed that maspin, like some other members of the serpin family, might act as an inhibitory serpin towards serine protease targets. Tissue-type plasminogen activator (tPA) and urokinase-type plasminogen activator (uPA) are two of such serine protease targets proposed (2). In endometrial cancer, a higher level of uPA and plasminogen activator inhibitor-I expression compared to normal endometrium, which increased with clinical staging, has been found (19). Hence, the plasminogen activator system may be an effector molecule involved though further exploration is awaited.
Expression of the maspin gene is thought to be regulated by both transcription factors as well as epigenetic events (2). In several cancer types, maspin gene expression was shown to be activated by wild-type p53, and inversely correlated with mutant p53 $(20,2)$. In endometrioid endometrial carcinoma, it has been reported that $\mathrm{p} 53$ mutation occurs in $\sim 40 \%$ of grade 3 cancers but only $3 \%$ of grade 1 and 2 cancers (21). The role of $\mathrm{p} 53$ in regulating maspin expression in endometrial tissue is hence not clear, but probably it is not an important regulator in this case. DNA methylation is proposed to be an important epigenetic regulatory mechanism of maspin expression, and promoter hypermethylation was found to be associated with silencing of maspin gene expression $(1,2)$. No report on this is available in endometrial cancer tissue and further study of this is certainly deserved.

In conclusion, we presented a preliminary study for the first time addressing the expression of maspin in endometrial cancer. The present study demonstrated that there is upregulated transcription of the maspin gene in endometrioid adenocarcinoma of endometrium, with concurrent nuclear and cytoplasmic expression. Further study is warranted to look into the underlying mechanisms and prognostic significance.

\section{Acknowledgements}

The authors would like to thank Dr Gary Tse for provision of frozen human breast tissue (positive control), Ms. Katherine Wong, Ms. Loucia Chan and Ms. Flora Tam for their technical support.

\section{References}

1. Schaefer JS and Zhang M: Role of maspin in tumor metastasis and angiogenesis. Curr Mol Med 3: 653-658, 2003.

2. Sheng S: The promise and challenge toward the clinical application of maspin in cancer. Front Biosci 9: 2733-2745, 2004.

3. Zou Z, Anisowicz A, Hendrix MJ, Thor A, Neveu M, Sheng S, Rafidi K, Seftor E and Sager R: Maspin, a serpin with tumorsuppressing activity in human mammary epithelial cells. Science 263: 526-529, 1994.

4. Domann FE and Futscher BW: Maspin as a molecular target for cancer therapy. J Urol 169: 1162-1164, 2003.

5. Maass N, Hojo T, Ueding M, Luttges J, Kloppel G, Jonat W and Nagasaki K: Expression of the tumor suppressor gene maspin in human pancreatic cancers. Clin Cancer Res 7: 812-817, 2001.

6. Sood AK, Fletcher MS, Gruman LM, Coffin JE, Jabbari S, Khalkhali-Ellis Z, Arbour N, Seftor EA and Hendrix MJC: The paradoxical expression of maspin in ovarian carcinoma. Clin Cancer Res 8: 2924-2932, 2002.

7. Li HWR, Leung SW, Cheung ANY, Yu MMY, Chan LKY and Wong YF: Expression of maspin in gestational trophoblastic disease. Gynecol Oncol 101: 76-81, 2006.

8. Umekita Y, Ohi Y, Sagara Y and Yoshida H: Expression of maspin predicts poor prognosis in breast cancer patients. Int $\mathrm{J}$ Cancer 100: 452-455, 2002.

9. Umekita $\mathrm{Y}$ and Yoshida $\mathrm{H}$ : Expression of maspin is upregulated during the progression of mammary ductal carcinoma. Histopathology 42: 541-545, 2003.

10. Mohsin SK, Zhang M, Clark GM and Craig AD: Maspin expression in invasive breast cancer: association with other prognostic factors. J Pathol 199: 432-435, 2003.

11. Ohike N, Maass N, Mundhenke C, Biallek M, Zhang M, Jonat W, Luttges J, Morohoshi T, Kloppel G and Nagasaki K: Clinicopathological significance and molecular regulation of maspin expression in ductal adenocarcinoma of the pancreas. Cancer Lett 199: 193-200, 2003. 
12. Son HJ, Sohn TS, Song SY, Lee JH and Rhee JC: Maspin expression in human gastric adenocarcinoma. Pathol Int 52: 508-513, 2002.

13. Bettstetter M, Woenckhaus M, Wild PJ, Rummele P, Blaszyk H, Hartmann A, Hofstadter F and Dietmaier W: Elevated nuclear maspin expression is associated with microsatellite instability and high tumour grade in colorectal cancer. J Pathol 205: 606-614, 2005.

14. Ogasawara S, Maesawa C, Yamamoto M, Akiyama Y, Wada K, Fujisawa K, Higuchi T, Tomisawa Y, Sato N, Endo S, Saito K and Masuda T: Disruption of cell-type specific methylation at the maspin gene promotor is frequently involved in undifferentiated thyroid cancers. Oncogene 23: 1117-1124, 2004.

15. Hirai K, Koizumi K, Haraguchi S, Hirata T, Mikami I, Fukusima M, Yamagishi S, Kawashima T, Okada D, Shimizu K and Kawamoto M: Prognostic significance of the tumor suppressor gene maspin in non-small cell lung cancer. Ann Thorac Surg 79: 248-253, 2005.

16. Lonardo F, Li X, Siddiq F, Singh R, Al-Abbadi M, Pass HI and Sheng S: Maspin nuclear localization is linked to favorable morphological features in pulmonary adenocarcinoma. Lung Cancer 51: 31-39, 2006.
17. Marioni G, Blandamura S, Giacomelli L, Calgaro N, Segato P, Leo G, Fischetto D, Staffieri A and de Filippis C: Nuclear expression of maspin is associated with a lower recurrence rate and a longer disease-free interval after surgery for squamous cell carcinoma of the larynx. Histopathology 46: 576-582, 2005.

18. Gima T, Kato H, Honda T, Imamura T, Sasazuki T and Wake N: DCC gene alteration in human endometrial carcinomas. Int $\mathbf{J}$ Cancer 57: 480-485, 1994.

19. Foca C, Moses EK, Quinn MA and Rice GE: Differential mRNA expression of urokinase-type plasminogen activator, plasminogen activator receptor and plasminogen activator inhibitor type-2 in normal human endometria and endometrial carcinomas. Gynecol Oncol 79: 244-250, 2000.

20. Zhang W and Zhang W: Tissue microarray analysis of maspin expression and its reverse correlation with mutant $\mathrm{p} 53$ in various tumors. Int J Oncol 20: 1145-1150, 2002.

21. Lax SF: Molecular genetic pathways in various types of endometrial carcinoma: from a phenotypical to a molecularbased classification. Virchows Arch 444: 213-223, 2004.

22. Schmittgen TD and Zakrajsek BA: Effect of experimental treatment on housekeeping gene expression: validation by realtime, quantitative RT-PCR. Biochem Biophys Methods 46: 69-81, 2000 . 